Unspezifischer Schwindel

\section{Den Patienten wieder ihr Gleichgewicht geben}

\author{
Schwindel ist in Haus- und Facharztpraxen nach dem Kopfschmerz ei- \\ nes der häufigsten Leitsymptome. Die Prävalenz von Schwindel liegt in \\ Abhängigkeit vom Alter zwischen 17\% bei Jüngeren und bis zu 33\% \\ bei den über 80-Jährigen. Die Lebenszeitprävalenz für mittelstarken \\ bis heftigen Schwindel wird mit nahezu 30\% angegeben.
}

Schwindel kann die Betroffenen in ihrem Alltag erheblich einschränken, berichtet Dr. Wolfgang Schwartz, niedergelassener Facharzt für Hals-Nasen-Ohrenheilkunde aus Braunschweig. Wer regelmäßig von solchen Störungen des Gleichgewichts heimgesucht wird, werde dadurch verunsichert und neige oftmals dazu, soziale Kontakte zu vermeiden. Schwindel beeinträchtige also den Alltag und die Lebensqualität ganz erheblich.

Frauen sind häufiger als Männer und Ältere öfter als Jüngere betroffen. Besonders multimorbide Patienten leiden vielfach unter Schwindel, vor allem weil sie Angst haben, zu stürzen und sich dabei zu verletzen. Tatsächlich sei das Risiko, dass es aufgrund der erhöhten Fallneigung zu einem Sturz mit Folgeschäden bis hin zur Hospitalisierung kommen kann, deutlich erhöht, betont Schwartz.
Grundlage der Diagnose seien eine eingehende Anamnese und der Ausschluss internistischer Ursachen. Im Gespräch sollte der Patient auch nach vorangegangenen Infekten gefragt werden, rät Schwartz. Flüssigkeitsmangel oder ungewohnte körperliche Belastungen können ebenfalls Schwindelsymptome verursachen. Viele Schwindelsyndrome lassen sich nach einer sorgfältigen Anamnese und körperlicher Untersuchung diagnostisch korrekt einordnen. Die Ursachen seien meist gutartig.

\section{Förderung der zerebralen Mikrozirkulation}

Dennoch bleibe immer noch ein bedenen keine relevanten vestibulären oder neurologischen Befunde vorliegen. Diese Patienten mit unspezifischem Schwindel seien in der Regel etwas älter. Bei ihstimmter Prozentsatz von Patienten, bei

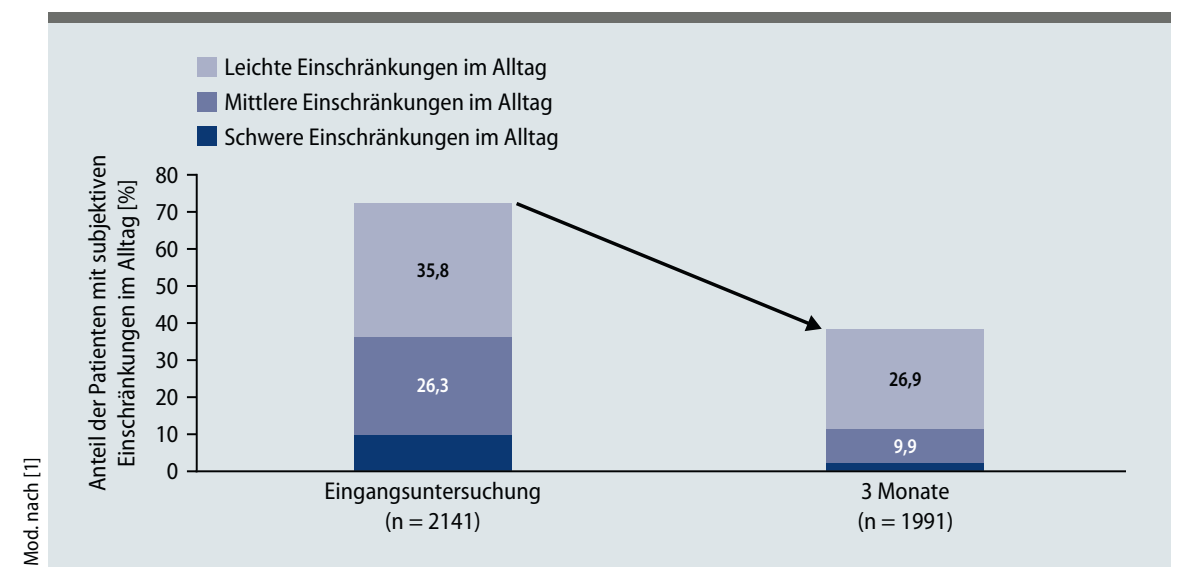

Abb. 1: Verringerung der schwindelassoziierten Alltagseinschränkungen. nen reduziere sich der Schwindel oftmals auf Durchblutungsstörungen etwa als Folge atherosklerotischer Prozesse, berichtet Schwartz. Im Hinblick auf die Förderung der zerebralen Mikrozirkulation sowie fehlender Nebenwirkungen und Interaktionen sollte in diesen Fällen eine Behandlung mit einem homöopathisch hergestellten Kombinationsarzneimittel wie Vertigoheel ${ }^{\triangleright}$ in Erwägung gezogen werden, empfiehlt Schwartz. Das Präparat wirkt von Anfang an und wird auf dem Grünen Rezept verordnet.

In einer großen, nicht-interventionellen Studie wurden Wirksamkeit und Verträglichkeit des homöopathischen Kombinationsarzneimittels bei älteren Patienten mit unspezifischem Schwindel untersucht [1]. In die Studie waren 2141 Patienten mit Schwindelsymptomen und Verdacht auf oder mit bekannter Arteriosklerose eingeschlossen. Zwei Drittel der Teilnehmer waren Patientinnen, das Durchschnittsalter lag bei 73 Jahren. Die überwiegende Zahl der Patienten klagte über unspezifische Schwindelsymptome wie Taumel, Gleichgewichtsprobleme oder Fallneigung.

\section{Im Alltag wieder}

besser zurechtkommen

In die Auswertung gingen die Daten von 1991 Patienten über die ersten drei Monate der Behandlung ein. Die Dosierung lag im Ermessen des Arztes. Beim Großteil der Patienten erfolgte die Therapie mit $3 \times 1$ bzw. 3 x 2 Tabletten täglich. Die größte Reduktion hinsichtlich Intensität, Anzahl und Dauer der Schwindelattacken konnte innerhalb der ersten drei Monate nach Beginn der Behandlung dokumentiert werden. Die allermeisten Patienten gaben nach diesem Zeitraum an, dass sie im Alltag wieder besser zurechtkommen (Abb. 1).

Über $80 \%$ der Ärzte beurteilten die Wirksamkeit der Dauertherapie mit dem homöopathischen Kombinationspräparat bei Patienten mit unspezifischen Schwindelsymptomen als gut bis sehr gut. Nach Abschluss der Studie waren die Ärzte und auch die Patienten mit der Behandlung insgesamt zufrieden. Sowohl Ärzte wie auch Patienten gaben an, dass sie aufgrund ihrer Erfahrungen Vertigoheel ${ }^{\circ}$ sehr wahrscheinlich erneut einnehmen bzw. verschreiben werden. 